\title{
Interactions between Ageing and Risk properties in the analysis of burn-in problems
}

\author{
Rachele Foschi \\ IMT Advanced Studies - Economics and Institutional Change Research Area, rachele.foschi@imtlucca.it, \\ Fabio Spizzichino \\ Università degli Studi di Roma La Sapienza, Dipartimento di Matematica, fabio.spizzichino@uniroma1.it,
}

\begin{abstract}
Several relevant problems in reliability can be looked at as problems of risk management and of decision in face of uncertainty. However, in this frame, the so-called burn-in problem can be seen as a problem of risk-taking par excellence. In this paper, we in particular point out some aspects concerning interactions between the probabilistic model for lifetimes and considerations of an economic kind.

As one of the features of our work, we hinge on some unexplored connections between ageing properties of a one-dimensional survival function $\bar{G}$ and risk-aversion type properties of the function $u(t)=b G(t), b>0$, when the latter is seen as a utility function.
\end{abstract}

Key words: Burn-in; Ageing; Utility functions; Risk

\section{Introduction}

In the reliability field, the term burn-in stands for the procedure of testing a new component for a period before its active life. This is a widely used engineering method to eliminate "weak" items from a population of industrial products, reducing the probability of early failures. One of the most widespread problems in this area is to decide how long such a procedure should last. The best time to stop the burn-in process for a given criterion is said the optimal burn-in time.

Such a stopping problem comes out as a completely natural one, taking into account the following circumstance: even in the cases when it is convenient performing a burn-in procedure, the duration of the latter should not be too long. In fact it is not convenient to wear out during the burn-in an item that could be used for operations; furthermore the burn-in procedure itself may be expensive in several situations.

This topic presents possible difficulties and ruses both of theoretical and "philosophical" type; in particular the terms "weak items" and "early failures" should constitute objects of deep reflections and cannot be taken as completely clear once for ever. For these reasons the related literature is really very wide. In this respect, we in particular address the reader to the technical papers and overview articles presented in the bibliography and to the references cited therein. In spite of the richness of the existing literature, we think that it may be of interest reflecting further upon this topic from a decision-analysis point of view.

Almost obviously, all the problems in reliability can be looked at as problems of decision in face of uncertainty and of risk management. However, the burn-in problem can be seen as a problem of risk-taking par excellence. In fact, the decision of stopping the burn-in and delivering a unit to operations can be directly interpreted as a bet under uncertainty conditions and consideration of risk concepts is completely natural in this frame.

In this paper, we want in particular to point out some aspects about interactions between the probabilistic model for lifetimes and considerations of an economic kind. As far as the probabilistic model is concerned, it is natural that properties of ageing (such as IFR, DFR, etc) of the marginal survival function $\bar{G}$ of the lifetime of an item enter in the burn-in problem. 
The criterion under which the optimal burn-in time has to be determined is not fixed a priori. It should correspond to a cost structure associated with the considered model. Several cost structures have been proposed in the literature; for a detailed survey, see e.g. Block and Savits (1997) and references therein.

We will consider here a reward function of the form

$$
R(t, \delta)=[\rho(t-\delta)+\tilde{k}] \mathbf{1}_{\{t \geq \delta\}}+k \mathbf{1}_{\{t<\delta\}}+c \min (t, \delta) .
$$

Thus, $R(t, \delta)$ depends on

- the burn-in time, $\delta>0$;

- the total lifetime of the unit, $t>0$ (burn-in time + time in operations);

As we will see in the following, $t$ will be the value taken by a random variable $T$ and $\delta$ is a quantity to be optimally determined.

The reward function consists of:

- a cost of conducting burn-in, $c \min (t, \delta)$, proportional to the actual burn-in time, by means of the constant cost rate $c<0$;

- a fixed cost due to failure during the burn-in, $k<0$;

- a fixed cost due to failure during the use, $\tilde{k}<0$;

- a reward $\rho:[0,+\infty) \rightarrow[0,+\infty)$, that is an increasing function of the duration of the unit in operations.

In order to avoid this optimization problem to be trivial, we assume $\tilde{k}<k<0$, i.e. the cost of losing the unit during burn-in is smaller than the cost of failure during operation.

However several are the versions of the problem we could obtain by modifying our reward function and many are the ones already considered in the literature, for modeling different kinds of usage of burned-in units.

As a main purpose of this work, we analyze the interactions between some ageing properties of $\bar{G}$ and qualitative properties of the function $\rho$ and their role in determining the optimal solution. Some more analytical aspects of this problem are studied in Foschi (2011).

Sometimes engineers may think that basic qualitative aspects of the optimal burn-in time for a component essentially depends on the ageing properties of its lifetime. In a Bayesian and a decisiontheory oriented approach, it is however well-established that a burn-in time (if any) heavily depends on both the properties of $G$ and of the reward function $R(t, \delta)$ (see e.g. the discussion presented in Lynn and Singpurwalla (1997) and references therein). The analysis developed in this paper aims at establishing some definite and detailed results in this direction, for reward functions of the specific form in (1). We believe however that our considerations can be substantially extended to other sensible reward structures, provided suitable adjustments are made.

A good part of the literature devoted to burn-in deals with bathtub-shaped failure-rate distributions (for a survey, see e.g. Lai and Xie (2006)). Such distributions model a non-negligible presence of early failures and, in this context, the problem of how long performing the burn-in naturally arises. In their practice, engineers may be led to conjecture that burn-in procedures are advisable only in the bathtub case or, at most, in cases that suitably generalize the bathtub condition (see Definition 1 and Remark 1 in the next section). However, when the model is endowed with a reward structure, both ageing of the lifetime distribution and analytical properties of the reward function concur to the determination of the optimal burn-in time (see again e.g. Lynn and Singpurwalla (1997)). We focus here on such interactions, without necessarily limiting our attention on the bathtub case. Actually we shall analyze in detail the existence of positive optimal burn-in times, even out of the bathtub case. 
In any case, the interactions between ageing and reward function may produce unexpected effects. In fact, within the bathtub case for instance, one may expect that the optimal burn-in time has necessarily to fall within the "initial" region of components' life, where $r$ is decreasing.

As a matter of fact, such a circumstance necessarily happen in the case when $\rho$ is linear, as we will show formally in Section 2. However there is no evident mathematical reason that it is still necessary when $\rho$ is non-linear. Concerning such cases (non-linear $\rho$ and bathtub or "definitively IFR" distributions), we will anyway obtain useful inequalities about optimal burn-in times as consequences of the results to be presented in Section 3.

As a specific feature of our paper, we show and subsequently use some unexplored connections between ageing and risk-aversion type properties of the function

$$
u(t)=b G(t), \quad b>0,
$$

that is associated with a one-dimensional survival function $\bar{G}(G=1-\bar{G})$ and that can be seen as a utility function. However, these connections will manifest in the formulations of our results concerning necessary (or, in some cases, necessary and sufficient) conditions for an instant $\delta>0$ to be an optimal burn-in time. Anyway, we believe that such connections may be of independent interest.

In the burn-in problem it is rather common to analyze the case of a single unit and we shall limit our attention here to such a case, as well. Notice that this restriction is completely justified even if we consider several units, provided that their lifetimes are stochastically independent. One could argue, however, that even the more complex case of several dependent units could be in a sense reduced to the case of several one-dimensional problems; in this respect see in particular Costantini and Spizzichino (1997), Jensen and Spizzichino (2004). A few more details in this respect can also be found within the discussion at the end of this paper.

The paper is organized as follows. In Section 1, we analyze, from a special viewpoint, some basic notions of ageing for a one-dimensional survival function $\bar{G}$ and introduce in the analysis the function $u$, that has been defined in Eq. (2) and whose properties turn out to be relevant; more precisely we point out some interactions between ageing and risk-aversion type properties of $u$. In Section 2, we analyze in detail the burn-in problem associated with a reward function of the kind in Eq. (1). This will put us in a position to discuss some effects, on the solution of the burn-in problem, of the relationships between ageing of $\bar{G}$ and risk-aversion properties in the case when $\rho$ is linear.

More precisely, we will see that a relation between hazard rate and risk aversion coefficient is needed as a necessary condition for a value $\delta$ to be an optimal burn-in time.

Section 3 is devoted to the extension of results of Section 2 to the case when $\rho$ is non-linear. We conclude the analysis of this case by comparing the plots of the expected rewards $\mathcal{R}(\delta) \equiv \mathbb{E}[R(T, \delta)]$ in different situations. Such comparisons are intended to exemplify our results about interactions among ageing properties of $G$, qualitative properties of $\rho$ and position of the optimal burn-in time. Finally, Section 4 is devoted to discussion and concluding remarks.

\section{Risk-aversion type characterizations of ageing properties}

In this section we analyze the link existing among ageing notions for non-negative random variables and properties of functions that will appear in the next Sections 2 and 3, along our study of the burn-in problem. On this purpose, we preliminarily point out some interactions between notions of ageing and notions of risk. 
Let $T$ be a random variable with survival function $\bar{G}$, probability distribution $G$ and density $g$. Its hazard rate is denoted by

$$
r(t):=r_{\bar{G}}(t)=\frac{g(t)}{\bar{G}(t)}=-\frac{\bar{G}^{\prime}(t)}{\bar{G}(t)} .
$$

We will assume $g$ to be differentiable as well, so that we can define, associated with $\bar{G}$, the function

$$
\alpha(t):=\alpha_{\bar{G}}(t)=-\frac{g^{\prime}(t)}{g(t)} .
$$

Consider now the following affine transformation of the survival function $\bar{G}$ :

$$
u(t)=b G(t)=-b \bar{G}(t)+b, \quad b>0 .
$$

The function $u$ can be seen as a utility function associated with $\bar{G}$ and we are then entitled to consider for it the local coefficient of risk aversion, as it was independently introduced by de Finetti and by Arrow and Pratt (see e.g. Scarsini (1994) and references cited therein). In this respect, it is interesting for our purposes to notice that we can write

$$
-\frac{u^{\prime \prime}(t)}{u^{\prime}(t)}=\alpha(t)
$$

where $\alpha$ is the function defined in Eq. (3). This interpretation will suggest us some links between ageing properties and risk.

For given $\bar{G}$ and $t>0$, we denote $\bar{G}_{t}(x) \equiv \frac{\bar{G}(t+x)}{\bar{G}(t)}$.

Also to the functions $\bar{G}_{t}$ we can associate their hazard rates $r_{t}$ and risk aversion coefficients $\alpha_{t}$. For what follows it is useful to preliminary notice that $r_{t}$ and $\alpha_{t}$ associated with $\bar{G}_{t}$ can be obtained just by translation:

$$
r_{t}(x)=r(t+x), \quad \alpha_{t}(x)=\alpha(t+x) .
$$

We recall that $\bar{G}$ is IFR (DFR) if $\bar{G}_{t}(x)$ is increasing (decreasing) in $t$ for any $x$.

Such ageing properties can be satisfied by a distribution $\bar{G}$, in a sense, only "from a certain time on". This issue suggests considering the following

Definition 1 . We say that, for a given $\delta>0, \bar{G}$ is $\delta$-IFR ( $\delta$-DFR) if $\bar{G}_{\delta}$ is IFR (DFR).

REMARK 1. The notion of $\delta$-IFR is a generalization of the bathtub shape, in the sense that a $\delta$-IFR distribution is definitively IFR, but, contrarily to what happens for a bathtub distribution, we do not know the monotonicity character of its hazard rate for $t<\delta$. Analogously, $\bar{G} \delta$-DFR, i.e. $\bar{G}$ definitively DFR, is a generalization of $\bar{G}$ upside down bathtub.

REMARK 2. It can be easily proven (see also Foschi and Spizzichino (2008)) that $\bar{G}_{\delta}$ IFR (DFR) is equivalent to each of the following properties

1. $\bar{G}_{t} \operatorname{IFR}(\mathrm{DFR})$ for any $t \geq \delta$;

2. $r(t)$ increasing (decreasing) for any $t \geq \delta$.

We point out that, obviously, for $\delta>0, \bar{G}$ IFR (DFR) is a stronger condition than $\bar{G} \delta$-IFR (resp. $\delta$-DFR) 
REMARK 3. By definition, the utility function $u(\delta)$ is such that $u(\delta)=b P(T \leq \delta)$ and we can, thus, give the following interpretation of it.

Consider the case of a large number $n$ of units, with i.i.d. lifetimes distributed according to $G$, and starting to work simultaneously. By the law of large numbers, $u(\delta)$ approximates the proportion $p(\delta)$ of units that failed before time $\delta$. It is reasonable in some situations to conceive that the utility of having a single unit surviving at $\delta$ is proportional to $p(\delta)$ : the less units are surviving, the more important becomes the availability of any single unit.

This interpretation can reveal as useful in reliability theory. In this respect, we notice that an interpretation of probability distribution functions as utility functions emerged in the frame of multi-attribute utilities. For example, Bordley and LiCalzi (2000) proposes a target-based approach for dealing with decision making. Such an approach, in some contexts, may turn out to be more fruitful than the traditional utility-based language. It consists in considering, in place of the classical utility function, the probability distribution of a target event. From this point of view, multiattribute utilities just turn out to be multivariate probability distributions.

The following result provides a link between an ageing property and a risk-aversion type property. Among its consequences (see Corollary 1), we will obtain conditions for $\bar{G}$ being IFR (or DFR) in terms of properties of a risk-aversion type necessary and sufficient.

Proposition 1. For any $t, r(t)$ is increasing at $t$ if and only if $r(t)>\alpha(t)$.

Proof. Let $r(t)$ be increasing at $t$. Then it must be

$$
r^{\prime}(t)=\frac{-\bar{G}^{\prime \prime}(t) \bar{G}(t)+\left[\bar{G}^{\prime}(t)\right]^{2}}{[\bar{G}(t)]^{2}}>0,
$$

i.e.

$$
-\bar{G}^{\prime \prime}(t) \bar{G}(t)>-\left[\bar{G}^{\prime}(t)\right]^{2} .
$$

Dividing both the sides by $\bar{G}(t) \bar{G}^{\prime}(t)<0$, we get

$$
\frac{-\bar{G}^{\prime \prime}(t)}{\bar{G}^{\prime}(t)}<\frac{-\bar{G}^{\prime}(t)}{\bar{G}(t)}
$$

that, by definition, coincides with $r(t)>\alpha(t)$.

Conversely, if $r(t)>\alpha(t)$, both the following alternatives are possible: $\alpha(t)<0$ or $\alpha(t)>0$. Let $\alpha(t)>0$, under the hypothesis $r(t)>\alpha(t)$, we reobtain $r^{\prime}(t)>0$. On the other hand, $\alpha(t)<0$ is equivalent to $\bar{G}^{\prime \prime}(t)<0$, which automatically implies $r^{\prime}(t)>0$.

Remark 4. As a consequence of Proposition 1, we can also claim that, for any $t, r(t)$ is decreasing at $t$ if and only if $r(t)<\alpha(t)$.

Remark 5. Notice that Proposition 1 provides only a point-wise condition (at $t$ ) and not a global condition such as the one of IFR.

For the developments to be presented in Section 2, we will use the following corollaries of Proposition 1.

Corollary 1. $\bar{G}$ is $\delta$-IFR ( $\delta$-DFR) if and only if $r(t)>\alpha(t)(r(t)<\alpha(t))$ for any $t \geq \delta$. 
In particular

Corollary 2. $\alpha(t)<0 \forall t \geq \delta \Rightarrow \bar{G} \delta$-IFR.

The relationship between $r$ and $\alpha$ has been already considered in Glaser (1980), Gupta (2001), giving conditions on $\alpha$ in order to establish the shape of $r$. We put ourselves in a different frame and perspective and obtain different results.

For functions $\sigma: \mathbb{R}^{+} \rightarrow \mathbb{R}$, let us define the function

$$
H_{\sigma}(\delta)=\int_{0}^{+\infty} \sigma(t)\left[\frac{g(t+\delta)}{g(\delta)}-\frac{\bar{G}(t+\delta)}{\bar{G}(\delta)}\right] d t .
$$

The latter function emerges in the following discussion. In particular, it is involved in Lemma 1, that will be used later on (see Section 3) for stating optimality conditions on a time $\delta$.

Lemma 1. Let $\sigma(t)<0$ for any $t \geq 0$ and let $\bar{G}$ be $\delta$-IFR for a given $\delta>0$. Then $H_{\sigma}(\delta)<0$.

Proof. By definition, $\bar{G}$ is $\delta$-IFR if and only if $r(\delta)<r(t+\delta)$ for any $t>0$.

By writing $r(t)$ as $\frac{g(t)}{\bar{G}(t)}$, we obtain the condition

$$
\frac{g(\delta)}{\bar{G}(\delta)}<\frac{g(t+\delta)}{\bar{G}(t+\delta)}
$$

and therefore

$$
\frac{g(t+\delta)}{g(\delta)}-\frac{\bar{G}(t+\delta)}{\bar{G}(\delta)}>0 .
$$

Hence, since the integrand is negative, $H(\delta)<0$.

\section{Properties of the burn-in time in the linear case}

Let $C$ be the component for which the burn-in problem is considered and let $T$ be its random lifetime. Let $\bar{G}$ denote the survival function of $T$ and refer, for the rest of notation, to the one introduced in Section 1.

Choosing the value $\delta$ for the burn-in time means that $C$ is tested for a time $\delta$. Then $C$ is put into operations only if it does not meet any failure in the time-interval $[0, \delta]$. Fixing $\delta=0$ obviously amounts to the choice of avoiding burn-in and putting directly $C$ into operations. Fixing $\delta>0$ means that the test period will last $\delta \wedge T=\min (\delta, T)$ and that the length of the operative life of $C$ will be $(T-\delta)^{+}=\max (0, T-\delta)$, i.e. we consider the decomposition

$$
T=(\delta \wedge T)+(T-\delta)^{+} .
$$

We denote by $R(t, \delta)$ the reward that we obtain if we fix the burn-in time equal to $\delta$ and we observe $\{T=t\}$. Choosing $\delta$ then becomes a decision problem in face of uncertainty and our optimization problem amounts to maximizing, with respect to $\delta$, the expected reward

$$
\mathcal{R}(\delta) \equiv \mathbb{E}[R(T, \delta)] .
$$

In this section, we first analyze in detail the burn-in problem associated with a specific reward function. Next we point out the role that the ageing properties and their interaction with risk have in determining the solution. To this purpose we define the criterion under which the optimum has to be determined. 
DeFinition 2. For a given reward function $R(t, \delta)$ and a random variable $T$, the optimal burn-in time $\delta^{*}$, is the point of absolute maximum for $\mathbb{E}[R(T, \delta)]$, i.e. the point such that

$$
\mathbb{E}\left[R\left(T, \delta^{*}\right)\right] \geq \mathbb{E}[R(T, \delta)] \text { for any } \delta \geq 0 .
$$

In the following we limit our analysis to the case where $R$ is of the form in Eq. (1), so that we have

$$
\mathcal{R}(\delta)=\tilde{k} \bar{G}(\delta)+k G(\delta)+\int_{0}^{+\infty} \rho(t) g(t+\delta) d t+c \delta \bar{G}(\delta)+c \int_{0}^{\delta} t g(t) d t .
$$

In this section and in the next one, we separately treat the cases where the function $\rho$ is linear (i.e. $\rho(t)=\rho_{0} t$, for some $\rho_{0}>0$ ) or non-linear.

In the latter case, special attention will be given to the assumption that $\rho$ is concave or convex.

We aim now at finding the optimal burn-in time, under our reward function.

To this purpose, the following reasonable assumption, about the probability distribution of $T$, will be used as a technical hypothesis in the proof of Proposition 2 below.

Assumption 1: $\lim _{x \rightarrow \infty} x g(x)=0$.

When $\rho$ is linear, let us consider the conditions

$$
\left\{\begin{array}{l}
r(\delta)=\frac{\rho_{0}-c}{k-\tilde{k}} \\
\alpha(\delta)>r(\delta)
\end{array}\right.
$$

Proposition 2. Let $\rho$ be linear. $\delta^{*}>0$ is a locally optimal burn-in time if and only if it satisfies the condition (6).

Proof. Maximizing the expected reward $\mathcal{R}(\delta)$ with respect to $\delta$ is equivalent to finding $\delta>0$ such that

As a first step, compute $\mathcal{R}^{\prime}(\delta)$ :

$$
\left\{\begin{array}{l}
\mathcal{R}^{\prime}(\delta)=0 \\
\mathcal{R}^{\prime \prime}(\delta)<0
\end{array}\right.
$$

$$
\mathcal{R}^{\prime}(\delta)=(k-\tilde{k}) g(\delta)+\rho_{0} \int_{0}^{+\infty} t g^{\prime}(t+\delta) d t+c \bar{G}(\delta) .
$$

By the linearity of $\rho$, implying $\rho(0)=0$,

$$
\int_{0}^{+\infty} t g^{\prime}(t+\delta) d t=-\int_{0}^{+\infty} g(t+\delta) d t=-\bar{G}(\delta) .
$$

Therefore, $\delta$ satisfies $\mathcal{R}^{\prime}(\delta)=0$ if and only if it satisfies

$$
r(\delta)=\frac{\rho_{0}-c}{k-\tilde{k}}
$$

Secondly, compute $\mathcal{R}^{\prime \prime}(\delta)$ :

$$
\mathcal{R}^{\prime \prime}(\delta)=\left(\rho_{0}-c\right) g(\delta)+(k-\tilde{k}) g^{\prime}(\delta) .
$$

Hence $\mathcal{R}^{\prime \prime}(\delta)<0$ if and only if $\alpha(\delta)>r(\delta)$. 
We can now apply the topics of the previous section to analyze further aspects of the solution of the burn-in problem. More precisely, we combine Proposition 2 with the arguments presented in Section 1.

The need for burn-in naturally arises in the presence of bathtub-shaped distributions. More generally, we can consider distributions that are definitively IFR, that is $\bar{\delta}$-IFR for a certain $\bar{\delta}$. In this respect, we have the following

Proposition 3. Let $\delta^{*}>0$ satisfy Eq. (7). If $\rho$ is linear and $\bar{G}$ is $\bar{\delta}$-IFR, then a necessary condition for $\delta^{*}$ being an optimal burn-in time is

$$
\delta^{*} \in[0, \bar{\delta}) .
$$

Proof. It follows from Proposition 2, by noticing that $\delta^{*}$ satisfying Eq. (7) is a maximum point only if it falls where $r$ is decreasing and therefore internally to the interval $[0, \bar{\delta})$.

Remark 6. Proposition 2, combined with Proposition 1, allows us to limit the search for the locally optimal points $\delta^{*}$ 's to the regions where $r$ is decreasing.

This fact leads us to the following conclusions. When $\bar{G}$ is $\bar{\delta}$-IFR (and not necessarily bathtub), we have no information on the monotonicity character of $r(\delta)$ for $\delta \in[0, \bar{\delta})$; therefore the condition $\delta^{*} \in\left[0, \bar{\delta}\right.$ ) (obviously, combined with Eq. (7)) is only necessary for the optimality of $\delta^{*}$.

In the particular case of bathtub-shaped distributions, $\delta^{*}<\bar{\delta}$ is a necessary and sufficient condition for the optimality of $\delta^{*}$.

In the even more particular case when $\bar{G}$ is IFR, the only admissible optimal burn-in time is $\delta^{*}=0$. Notice that the restriction for the optimal burn-in time to fall within the regions where $r$ is decreasing here follows formally from the assumption that $\rho$ is linear. We point out, as a matter of fact, that such a restriction is not automatic in general, even if it may appear obvious from an intuitive view-point.

We highlight however that strictly positive optimal burn-in times can be found out of the definitively IFR case. In such cases, we can find even more explicit conditions for optimality. In this respect, Theorem 1 below puts us in a position to obtain more transparent conditions than those given by Proposition 2 .

The link between Proposition 2 and Theorem 1 is given by the following Proposition 4 .

Proposition 4. Let $\rho$ be linear and $\bar{G}$ be $\bar{\delta}$-DFR. Then $\mathcal{R}^{\prime \prime}(\delta)<0$ for any $\delta \geq \bar{\delta}$.

Proof. By Proposition 1 and its Corollary 1, $\bar{G} \bar{\delta}$-DFR implies $r(\delta)<\alpha(\delta)$ for any $\delta \geq \bar{\delta}$, that, in the linear case, is an equivalent condition to $\mathcal{R}^{\prime \prime}(\delta)<0$ for any $\delta \geq \bar{\delta}$.

The following theorem provides sufficient ageing conditions for the optimality of a burn-in time.

Theorem 1. Let $\rho$ be linear. If $\delta^{*}>0$ exists such that

- $r\left(\delta^{*}\right)=\frac{\rho_{0}-c}{k-\tilde{k}}$,

- $\bar{G}$ is $\delta^{*}-D F R$,

then $\delta^{*}$ is a locally optimal burn-in time.

Furthermore, no locally optimal times fall in $\left(\delta^{*},+\infty\right)$. 
Proof. The first part straightly follows from Propositions 2 and 4.

Since in $\left(\delta^{*},+\infty\right) r$ is decreasing, for $\delta \in\left(\delta^{*},+\infty\right) r(\delta)<r\left(\delta^{*}\right)=\frac{\rho_{0}-c}{k-\tilde{k}}$ and therefore the equality $r(\delta)=\frac{\rho_{0}-c}{k-\tilde{k}}$ is no more satisfied.

REMARK 7. It can be of general interest finding the conditions for a value $\delta>0$ to perform better than 0 , as a possible a burn-in time, i.e. $\mathcal{R}(\delta)>\mathcal{R}(0)$. In view of Eq. (5), such inequality is equivalent to

$$
(k-\tilde{k}) G(\delta)+\int_{0}^{+\infty} \rho(t)[g(t+\delta)-g(t)] d t+c\left[\delta \bar{G}(\delta)+\int_{0}^{\delta} t g(t) d t\right]>0 .
$$

Along the whole paper, we neglect to consider situations where the optimal burn-in time may be zero. In fact, such an optimum cannot be detected by checking (6) (or (11) below), in that $\mathcal{R}$ is not differentiable in $\delta=0$. Hence, once we found $\delta^{*} \in(0,+\infty)$ by means of the conditions (6), in order to have that $\delta^{*}$ is the point of optimum, still we have to check that $\mathcal{R}\left(\delta^{*}\right)>\mathcal{R}(0)$. If such a $\delta$ satisfying (6) does not exist, we can put $\delta^{*}=0$.

To sum up, we showed how ageing properties of $\bar{G}$ and the form of the reward function interact in determining the value of the optimal burn-in time. In particular, Theorem 1 tells us that the DFR property of $\bar{G}_{\delta}$, in order to be a sufficient condition for $\delta$ being the optimum burn-in time, must be satisfied concurrently with the condition

$$
r(\delta)=\frac{\rho_{0}-c}{k-\widetilde{k}},
$$

which involves $\rho_{0}$ and the cost parameters $\tilde{k}, k, c$.

\subsection{Discussion and figures}

Here we want to illustrate more in detail how both ageing properties of $\bar{G}$ and the form of $R$ (that, in the present linear case, is completely determined by fixing $\rho_{0}, \tilde{k}, k, c$ ) influence the position of the optimal burn-in time.

Preliminarily, in order to display the graph of $\mathcal{R}(\delta)$, we have to suitably choose the cost parameters $\tilde{k}, k, c$. In their choice, some constrains must be respected. First of all, the expected reward must be greater than zero at least for some value $\delta$. It is obvious in fact that, if $\mathcal{R}$ were everywhere negative, it would not be convenient even to start producing a certain item. A sufficient condition for

$$
\exists \delta \geq 0 \text { s.t. } \mathcal{R}(\delta)>0
$$

is

$$
\mathcal{R}(0)>0, \quad \text { i.e. } \quad \tilde{k}>-\mathbb{E}[\rho(T)] .
$$

Notice that the latter condition is much easier to be checked than (8). In particular, for $\rho$ linear, it further simplifies in

$$
\tilde{k}>-\rho_{0} \mathbb{E}[T] .
$$

In order to solve a system with three unknowns, (at least) three constrains are of course needed. Therefore, in order to determine $k$ and $c$, we rely on the following two conditions:

1. (7) admits solution for some $\delta>0$;

2. $k>\tilde{k}+\frac{\rho-c}{g(0)}$. 
The latter condition is derived from $\mathcal{R}^{\prime}(0)>0$. In fact, in the linear case, it can be shown that, if $\mathcal{R}$ is decreasing in 0 , the burn-in will not take place at all. A more formal statement and a related proof can be found in (Foschi 2011, Proposition 1). Therefore, we must reject all the couples $(k, c)$ yielding $\mathcal{R}^{\prime}(0)<0$. Hence, the admissible values for $(k, c)$ are given by the inequality

$$
k>\tilde{k}+\frac{\rho-c}{g(0)} .
$$

In Fig. 1, we show how the choice of the cost parameters affects the shape of $\mathcal{R}$, by focusing in particular on the consequences of choosing "non-admissible" triples. At this purpose, we compare the functions $\mathcal{R}$ corresponding to the following cost parameters:

- $(\tilde{k}, k, c)=(-0.2,-0.03,-0.0036)$ (in red),

- $(\tilde{k}, k, c)=(-0.15,-0.003,-0.05)$ (in green),

- $(\tilde{k}, k, c)=(-0.2,-0.1,-0.005)$ (in blue).

Following the statement of Theorem 1, we fix the DFR distribution function

$$
G(t)=1-p e^{-\lambda_{1} t}-(1-p) e^{-\lambda_{2} t},
$$

with $p=0.3, \lambda_{1}=5, \lambda_{2}=15$, and $\rho(t)=2 t$.

The choice of a DFR distribution allows us to better point out the onset of local maxima, since for such distributions we have more usable conditions for determining them.

Figure 1 Comparison among expected rewards associated with same DFR $G$ and linear $\rho$ and different cost parameters

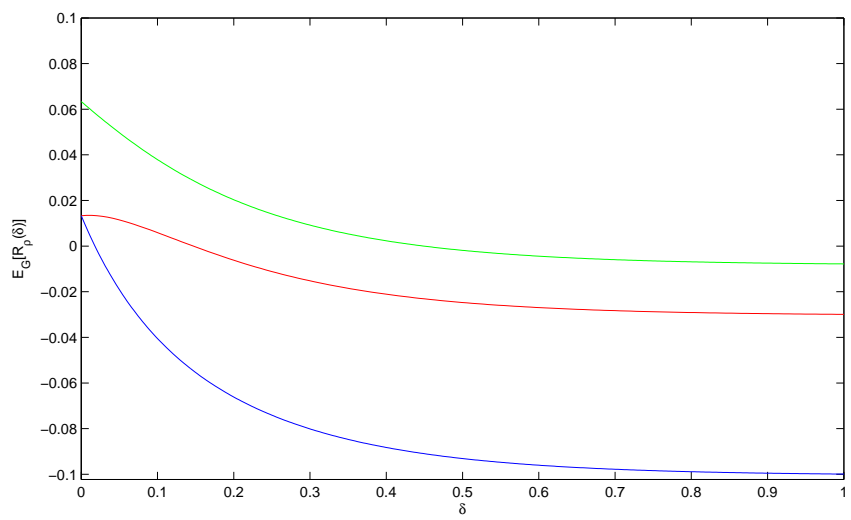

The first triple is the only admissible one, that is the only one satisfying all the three constrains provided above. In fact, the red curve is the only one admitting a maximum point different from 0 , namely $\delta^{*}=0.01$. The other curves admit no local maxima; therefore the associated optimal burn-in time is $\delta^{*}=0$.

The green curve is the one staying for the longest time above 0, i.e. the associated cost structure allows a non-optimal, but positive, reward also on the right of $\delta^{*}=0$. Such a longer positiveness allows a greater margin of error than in the other cases. The green curve in fact corresponds to the greatest value for the parameter $k$, which has the meaning of asymptotic expected reward.

We also notice that we cut the burn-in time axis at $\delta=1$. At that point $\mathcal{R}$ starts to be in a good approximation constant, since the survival probability of the item is $\bar{G}(1)=0.002$.

In Fig. 2, we consider the triple giving a maximum in the previous figure, $(\tilde{k}, k, c)=$ $(-0.2,-0.03,-0.0036)$, and compare the expected rewards associated with different distributions $G$ with a same mean value $\mathbb{E}(T)=\frac{8}{75}$, but different ageing properties: 
a) $G(\delta)=\frac{\beta^{\alpha}}{\Gamma(\alpha)} \int_{0}^{\delta} x^{\alpha-1} e^{-\beta x} d x$ (in red),

b) $G(\delta)=1-p e^{-\lambda_{1} \delta}-(1-p) e^{-\lambda_{2} \delta}$ (in green),

c) $G(\delta)=1-e^{-\lambda \delta}$ (in blue);

a) is a Gamma, that, for $\alpha>1$, is IFR;

b) is a mixture of exponential distributions, that is DFR;

c) is an exponential, having constant failure rate, therefore no ageing.

We choose the parameters $\alpha=2$, in order to make the gamma distribution IFR, $\lambda_{1}=5, \lambda_{2}=15$, $p=0.3$; since we want the three distributions to have a same mean, the remaining parameters are determined as

$$
\lambda=\frac{\lambda_{1} \lambda_{2}}{\lambda_{1}(1-p)+p \lambda_{2}}, \quad \beta=\alpha \lambda
$$

Figure 2 Comparison among expected rewards associated with a same linear $\rho$ and different $G$ 's

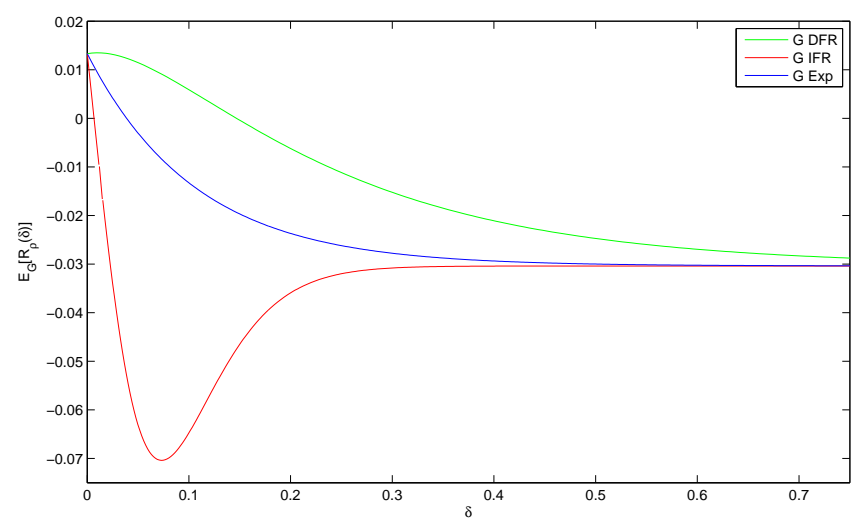

The three expected rewards take about the same value in $\delta=0$. Coherently with Theorem 1 , the following happens:

- for $G$ exponential, $\mathcal{R}$ is strictly decreasing;

- for $G$ DFR, $\mathcal{R}$ admits a maximum point greater than 0 ;

- for $G$ IFR, we observe a minimum.

The last point is coherent with Remark 6 , for which, since $\rho$ is linear and $G$ is IFR (i.e. $\bar{\delta}$-IFR with $\bar{\delta}=0$ ), the optimal burn-in time has to fall in $[0, \bar{\delta})$.

\section{Extension to the non-linear case}

As we have seen in the previous section, the analysis of optimality of burn-in is, mathematically, rather tractable in the case when the function $\rho$ is linear. The present section is devoted to the case when $\rho$ is not linear. In such a case we can still obtain, along the same line, some results, even if weaker than those valid for the linear case. Special cases of interest are met when $\rho$ is concave or convex.

In view of our results, it will be convenient to assume the following conditions, that are completely justified from a modeling point of view. 
- Assumption 2: $\rho(0)=0$;

- Assumption 3: $\lim _{x \rightarrow \infty} \rho^{\prime \prime}(x)=0$.

Proposition 5. $\rho$ concave implies Assumption 3.

Proof. Since $\rho$ is a reward function, $\rho^{\prime}(x) \geq 0$ for any $x$, but, since $\rho$ is concave, one must also have $\rho^{\prime}(x)$ decreasing for any $x$. This implies $\lim _{x \rightarrow+\infty} \rho^{\prime}(x)=b \geq 0$, that, on its turn, implies $\lim _{x \rightarrow+\infty} \rho^{\prime \prime}(x)=0$.

We are also interested in some analytical consequences of Assumption 3 (see Eq.'s (9) and (10)), that are needed as technical hypotheses in the proof of the Theorem 2 below.

Proposition 6. Assumption 3 implies

$$
\lim _{x \rightarrow \infty} \rho(x) g(x)=0 .
$$

Assumptions 1 and 3 imply

$$
\lim _{x \rightarrow \infty} \rho^{\prime}(x) \bar{G}(x)=0 .
$$

Proof. From Assumption 3, it follows that $\rho(x)$ is asymptotically linear, i.e., for $x$ going to infinity, $\rho(x) \approx a x$, for some $a>0$ (we can write $\lim _{x \rightarrow+\infty} \rho(x)=\lim _{x \rightarrow+\infty} a x$ ). Thus $\lim _{x \rightarrow+\infty} \rho^{\prime}(x)=a$ and it straightly follows that

$$
\lim _{x \rightarrow+\infty} \rho^{\prime}(x) \bar{G}(x)=0 .
$$

Again by Assumption 3 and in view of Assumption 1, we also obtain

$$
\lim _{x \rightarrow+\infty} \rho(x) g(x)=\lim _{x \rightarrow+\infty} \operatorname{axg}(x)=0 .
$$

Theorem 2 below provides us with a criterion for determining locally optimal burn-in times when $\rho$ is not linear.

Once we found such locally optimal points, the global maximum can be found by means of the standard procedure, i.e. we compare the values taken by the expected reward $R(\delta)$ in the formerly detected points and choose the value $\delta$ yielding the greatest value of $R(\delta)$.

Thus, like Proposition 2, the following result (Theorem 2) gives a sufficient and necessary condition for locally optimal points and only provides a necessary condition for $\delta^{*}$ being the global optimum.

Conditions (6), that hold in the linear case, can be extended to the non-linear case in the following way:

where

$$
\left\{\begin{array}{l}
r(\delta)=\frac{\mathbb{E}\left[\rho^{\prime}(T-\delta) \mid T>\delta\right]-c}{k-\tilde{k}} \\
\alpha(\delta)>r(\delta)+K(\delta)
\end{array}\right.
$$

i.e.

$$
K(\delta)=K_{\rho}(\delta)=\frac{H_{\rho^{\prime \prime}}(\delta)}{k-\tilde{k}},
$$

$$
K(\delta)=\frac{\int_{0}^{+\infty} \rho^{\prime \prime}(t)\left[\frac{g(t+\delta)}{g(\delta)}-\frac{\bar{G}(t+\delta)}{\bar{G}(\delta)}\right] d t}{k-\tilde{k}} .
$$

We can, at this point, generalize Proposition 2 as follows. 
THEOREM 2. $\delta^{*}>0$ is a locally optimal burn-in time if and only if it satisfies (11).

Proof. Maximizing the expected reward $\mathcal{R}(\delta)$ is equivalent to finding the value $\delta \geq 0$ that satisfies the conditions

$$
\left\{\begin{array}{l}
\mathcal{R}^{\prime}(\delta)=0 \\
\mathcal{R}^{\prime \prime}(\delta)<0
\end{array}\right.
$$

Compute $\mathcal{R}^{\prime}(\delta)$ :

$$
\mathcal{R}^{\prime}(\delta)=(k-\tilde{k}) g(\delta)+\int_{0}^{+\infty} \rho(t) g^{\prime}(t+\delta) d t+c \bar{G}(\delta) .
$$

Integrating by parts,

$$
\int_{0}^{+\infty} \rho(t) g^{\prime}(t+\delta) d t=[\rho(t) g(t+\delta)]_{0}^{+\infty}-\int_{0}^{+\infty} \rho^{\prime}(t) g(t+\delta) d t
$$

On its turn, the right-hand integral can be expressed both as

$$
\int_{0}^{+\infty} \rho^{\prime}(t) g(t+\delta) d t=\mathbb{E}\left[\rho^{\prime}(T-\delta) \mid T>\delta\right] \bar{G}(\delta)
$$

or, by integrating by parts a second time,

$$
\int_{0}^{+\infty} \rho^{\prime}(t) g(t+\delta) d t=\left[\rho^{\prime}(t) \bar{G}(t+\delta)\right]_{0}^{+\infty}-\int_{0}^{+\infty} \rho^{\prime \prime}(t) \bar{G}(t+\delta) d t .
$$

Therefore

$$
\int_{0}^{+\infty} \rho(t) g^{\prime}(t+\delta) d t=-\mathbb{E}\left[\rho^{\prime}(T-\delta) \mid T>\delta\right] \bar{G}(\delta)=-\rho^{\prime}(0) \bar{G}(\delta)-\int_{0}^{+\infty} \rho^{\prime \prime}(t) \bar{G}(t+\delta) d t .
$$

$\mathcal{R}^{\prime}(\delta)=0$ is thus equivalent to

$$
(k-\tilde{k}) r(\delta)-\int_{0}^{+\infty} \rho^{\prime \prime}(t) \frac{\bar{G}(t+\delta)}{\bar{G}(\delta)} d t-\rho^{\prime}(0)+c=0,
$$

that is

$$
r(\delta)=\frac{\mathbb{E}\left[\rho^{\prime}(T-\delta) \mid T>\delta\right]-c}{k-\tilde{k}} .
$$

Secondly, compute $\mathcal{R}^{\prime \prime}(\delta)$ :

$$
\mathcal{R}^{\prime \prime}(\delta)=\left(\rho^{\prime}(0)-c\right) g(\delta)+(k-\tilde{k}) g^{\prime}(\delta)+\int_{0}^{+\infty} \rho^{\prime \prime}(t) g(t+\delta) d t .
$$

$\mathcal{R}^{\prime \prime}(\delta)<0$ if and only if

$$
\alpha(\delta)>\frac{\int_{0}^{+\infty} \rho^{\prime \prime}(t) \frac{g(t+\delta)}{g(\delta)} d t+\rho^{\prime}(0)-c}{k-\tilde{k}},
$$

that is

$$
\alpha(\delta)>r(\delta)+K(\delta)
$$

with $K$ defined in (12). 
We point out that Proposition 2 could have been simply obtained as a particular case of Theorem 2. On the other hand, the linear case may be used as reference point for the development of the other cases. Then we preferred to treat it first and to provide an independent proof.

A further particular instance of relevance of Theorem 2 is the one when $\bar{G}$ has no ageing.

In this case, the optimal solution is independent of $\rho$.

Corollary 3. Let $\bar{G}$ be exponential. Then $\delta^{*}=0$.

Proof. Let $\bar{G}(\delta)=e^{-\lambda \delta}$, for some $\lambda>0$. Then $K(\delta)=0$ and Eq. (14) reduces to $\alpha(\delta)>r(\delta)$, as in the linear case. This last inequality is never satisfied, since, for any $\delta>0$,

$$
\alpha(\delta)=r(\delta)=\lambda
$$

Moreover, being $r$ constant, two situations are possible:

- $r$ satisfies Eq. (13) for any $\delta$, in which case, the whole half-line is a set of locally extremal points for $\mathcal{R}$ getting the same value for $\mathcal{R}$;

- $r$ never satisfies Eq. (13), in which case, the optimum does not exist.

In both the cases, we get $\delta^{*}=0$.

The following lemma allows us to obtain some links between asymptotic ageing properties and the position of the optimal burn-in time, depending on the shape of $\rho$ being concave or convex.

Lemma 2. Let $\rho$ be concave and $\bar{G} \delta$-IFR (or $\rho$ convex and $\bar{G} \delta$-DFR) for some $\delta$. Then $K(\delta)<0$. Let $\rho$ be concave and $\bar{G} \delta$-DFR (or $\rho$ convex and $\bar{G} \delta$-IFR) for some $\delta$. Then $K(\delta)>0$.

Proof. Notice that $k-\tilde{k}>0$ is a constant and that $\rho$ being concave implies $\rho^{\prime \prime}(t)<0$ for any $t$. Since $K_{\rho}(\delta)=\frac{H_{\rho^{\prime \prime}}(\delta)}{k-\tilde{k}}$, we are in the hypotheses of Lemma 1 and the thesis follows.

The proof of the other cases follows an analogous reasoning.

In the case when $\rho$ is convex, we obtain the following results, that, along the line of the ones holding in the linear case, are distinct for $\bar{\delta}$-IFR and $\bar{\delta}$-DFR distributions.

Proposition 7. Let $\delta^{*}>0$ satisfy Eq. (13). If $\rho$ is convex and $\bar{G}$ is $\bar{\delta}-I F R$, then a necessary condition for $\delta^{*}$ being an optimal burn-in time is

$$
\delta^{*} \in[0, \bar{\delta}) .
$$

Proof. It follows from Theorem 2, by noticing that $\delta^{*}$ satisfying Eq. (13) is a maximum point only if it also satisfies Eq. (14). But, on one hand, by Corollary $1, \bar{G} \bar{\delta}$-IFR implies $\alpha(\delta)<r(\delta)$ for any $\delta \geq \bar{\delta}$; on the other hand, by Lemma 2 , for any $\delta \geq \bar{\delta}, K(\delta)>0$. Hence, since $\rho$ convex and $\bar{G}$ $\bar{\delta}$-IFR imply $\alpha(\delta)<r(\delta)+K(\delta)$ for any $\delta \geq \bar{\delta}$, Eq. (14) can be satisfied only in the interval $[0, \bar{\delta})$.

The following theorem considers the case when $\bar{G}$ is $\bar{\delta}$-DFR. Notice that, in this case, it provides a sufficient condition for the optimality of $\delta^{*}$.

TheOREm 3. Let $\rho$ be convex and $\bar{G}$ be $\delta$-DFR for some $\delta$. The condition

$$
r\left(\delta^{*}\right)=\frac{\mathbb{E}\left[\rho^{\prime}\left(T-\delta^{*}\right) \mid T>\delta^{*}\right]-c}{k-\tilde{k}}
$$

for some $\delta^{*} \geq \delta$, implies that $\delta^{*}$ is a locally optimal burn-in time. 
Proof. Since $\bar{G}$ is $\delta$-DFR, it follows that $\alpha(t)>r(t)$ for any $t \geq \delta$. The further assumption of convexity of $\rho$ implies that, for any $t \geq \delta, K(t)<0$. Hence, we have $\alpha(t)>r(t)+K(t)$ for any $t \geq \delta$. The thesis follows by Theorem 2 .

As to the concave case, we will discuss in the following remark the ageing situations that may occur.

To this purpose we define the two sets $\mathcal{D}$ and $\mathcal{I}$ as follows:

$$
\mathcal{D}:=\{\delta>0 \mid \bar{G} \delta \text {-DFR }\}
$$

and define $\widehat{\delta}$ by

with $\widehat{\delta}=\infty$ if $\mathcal{D}=\emptyset$;

$$
\widehat{\delta}:=\inf \mathcal{D}
$$

$$
\mathcal{I}:=\{\delta>0 \mid \bar{G} \delta \text {-IFR }\}
$$

and

with $\bar{\delta}=\infty$ if $\mathcal{I}=\emptyset$.

$$
\bar{\delta}:=\inf \mathcal{I}
$$

The condition $\mathcal{D}=\emptyset$ occurs when a $\delta^{\prime}$ exists such that $\bar{G}$ is $\delta$-IFR or when, for any $t_{0}>0$, a $\delta^{\prime}>t_{0}$ exists such that $r^{\prime}\left(\delta^{\prime}\right)=0$, i.e. when $r$ changes its monotonicity infinitely many times and therefore, for any $\delta, \bar{G}$ is neither $\delta$-IFR nor $\delta$-DFR.

We notice that at least one between the two sets $\mathcal{D}$ and $\mathcal{I}$ must be empty.

REMARK 8. Consider the conditions $\alpha(\delta)>r(\delta)$ and $\alpha(\delta)>r(\delta)+K(\delta)$, respectively appearing in the statements of Proposition 2 and Theorem 2. These are only point-wise conditions and, as such, they do not imply that $\mathcal{D} \neq \emptyset$ (which is, actually, a global condition). In this respect, one might guess that $\delta^{*}$ being a locally optimal burn-in time implies, in any case, at least some local condition on the monotonicity-like behaviour of the hazard rate $r(t)$ at $\delta^{*}$.

In the non-linear case, this guess is not necessarily correct. Let us come back in fact to Theorem 2. The condition required to have a point of optimum in $\delta^{*}$ is $\alpha\left(\delta^{*}\right)>r\left(\delta^{*}\right)+K\left(\delta^{*}\right)$. To fix the ideas, let us assume $\rho$ to be concave.

If we have $r^{\prime}\left(\delta^{*}\right)>0$, Proposition 1 implies $\alpha\left(\delta^{*}\right)<r\left(\delta^{*}\right)$ and therefore, for $\alpha\left(\delta^{*}\right)>r\left(\delta^{*}\right)+K\left(\delta^{*}\right)$ being satisfied, it is necessary that $K\left(\delta^{*}\right)<0$. On its turn, the last condition implies, by Lemma 2 , that $r$ cannot be everywhere increasing; in particular a time $s>0$ exists, such that $r^{\prime}\left(s+\delta^{*}\right)<0$. This means that somewhere in the region $\left(\delta^{*},+\infty\right), r$ must change its monotonicity character at least once, that is, the following alternatives may occur:

- $\bar{G}$ is $\delta$-DFR, for some $\delta \geq s+\delta^{*}$ (i.e. $\mathcal{D} \neq \emptyset$ and $\mathcal{I}=\emptyset$ );

- $\bar{G}$ is $\delta_{0}$-IFR, for some $\delta_{0}>s+\delta^{*}$ (i.e. $\mathcal{D}=\emptyset$ and $\mathcal{I} \neq \emptyset$ );

- $r^{\prime}$ changes its sign infinitely many times (i.e. $\mathcal{D}=\mathcal{I}=\emptyset$ ).

If instead we have $r^{\prime}\left(\delta^{*}\right)<0$, Proposition 1 implies $\alpha\left(\delta^{*}\right)>r\left(\delta^{*}\right)$ and therefore $\alpha\left(\delta^{*}\right)>r\left(\delta^{*}\right)+K\left(\delta^{*}\right)$ is implied by $K\left(\delta^{*}\right)<0$. On its turn, this last condition is implied by $\bar{G}$ being $\delta$-IFR, for some $\delta \leq \delta^{*}$, in contradiction with the hypothesis $r^{\prime}\left(\delta^{*}\right)<0$. A weaker ageing condition on $\bar{G}$, consistent with $r^{\prime}\left(\delta^{*}\right)<0$, is $\bar{G}$ being $\left(s+\delta^{*}\right)$-IFR, for any $s>0$; it implies $K\left(\delta^{*}+s\right)<0$ for any $s>0$ and hence, by $K^{\prime}$ 's continuity, $K\left(\delta^{*}\right)<0$. Therefore, when $r^{\prime}\left(\delta^{*}\right)<0$, the following situations may arise:

- $\bar{G}$ is $\delta$-DFR, for some $\delta \leq \delta^{*}$;

- $\bar{G}$ is $\left(s+\delta^{*}\right)$-IFR, for any $s>0$;

- $r^{\prime}$ changes its sign infinitely many times.

Let us come back to the guess that $\delta^{*}$ being a locally optimal burn-in time implies some ageing condition on $\bar{G}$. On the basis of the above arguments, we can conclude that this guess in not correct. In fact the existence of an optimum does not univocally imply a monotonicity condition on $r$, rather a series of different alternatives is possible. 


\subsection{Discussion and figures}

After treating some theoretical aspects of the case when $\rho$ is non-linear, here we perform some graphical comparisons between the linear case and the non-linear one. In this more general case, of course, the form of $R$ is determined not only by $\rho^{\prime}(0), \tilde{k}, k, c$, but also by the shape of $\rho$.

Thus, along the line of what has been done in the linear case, we present here some examples of how the shape of $\rho$ influences the onset of extremal points for $\mathcal{R}$.

In particular, in the case when $\rho$ is concave, we can repeat what we did in Subsection 2.1. Namely, we consider the interactions between ageing properties of $\bar{G}$ and the form of $R$ in determining the position of the optimal burn-in time.

Now we turn to consider the following functions:

- $\rho(t)=\rho_{l}(t)=2 t$;

- $\rho(t)=\rho_{c v}(t)=\sqrt{4 t+1}-1$;

- $\rho(t)=\rho_{c x}(t)=2 t+t^{2}$.

These functions, that are respectively linear, concave and convex, have been selected so to share the following two properties:

- $\rho(0)=0$

- $\rho_{0} \equiv \rho_{l}^{\prime}(0)=\rho_{c v}^{\prime}(0)=\rho_{c x}^{\prime}(0)$.

In the present case, $\rho_{0}=2$.

In Fig. 3, we analyze the concave case $\rho(t)=\rho_{c v}(t)$ and, as to the distribution of the lifetime, we consider a mixture of exponentials. More precisely we take

$$
G(t)=1-p e^{-\lambda_{1} t}-(1-p) e^{-\lambda_{2} t},
$$

with $p=0.3, \lambda_{1}=2, \lambda_{2}=5$, and a concave $\rho(t)=\sqrt{4 t+1}-1$.

Figure 3 Comparison among expected rewards associated with same DFR $G$ and concave $\rho$ and different cost parameters

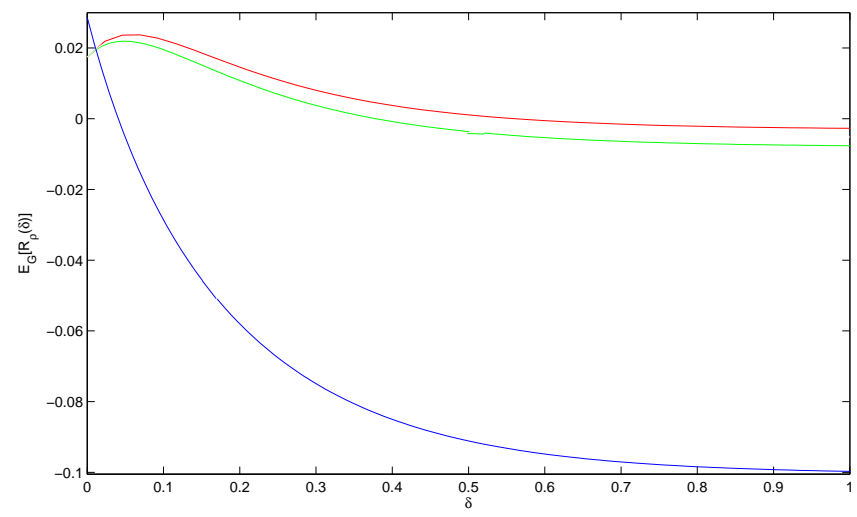

We then compare the graphs that are obtained for the expected reward $\mathcal{R}$ under the following different choices for the cost parameters (i.e. the same as we did for the linear case in Subsection 2.1):

- $(\tilde{k}, k, c)=(-0.2,-0.03,-0.0036)$ (in red),

- $(\tilde{k}, k, c)=(-0.15,-0.003,-0.05)$ (in green), 
- $(\tilde{k}, k, c)=(-0.2,-0.1,-0.005)$ (in blue).

We notice that, here, we obtain a strictly positive maximum point in correspondence to both the triples $(\tilde{k}, k, c)=(-0.2,-0.03,-0.0036)$ and $(\tilde{k}, k, c)=(-0.15,-0.003,-0.05)$. We consider therefore these two triples as admissible cost parameters.

Since $\rho$ may be here concave or convex, we cannot rely any more on the necessary and sufficient conditions given by Theorem 1 for the case when $\rho$ is linear. The present case is however covered by Theorem 3. Provided that the condition (15) is satisfied at $\delta^{*}$, such a result says that $\bar{G}$ being $\delta$-DFR is only a sufficient condition for $\delta^{*}$ being a point of local optimum.

The problem remains to understand how ageing properties of $G$ affect the shape of $\mathcal{R}$ (and hence the position of its extremal points).

In this respect we present a graphical example in Fig. 4 below. By fixing $(\tilde{k}, k, c)=$ $(-0.2,-0.03,-0.0036)$, we compare the expected rewards associated with different distributions $G$ with a same mean value, but different ageing properties; more precisely:

a) $G(\delta)=\frac{\beta^{\alpha}}{\Gamma(\alpha)} \int_{0}^{\delta} x^{\alpha-1} e^{-\beta x} d x$ (in red),

b) $G(\delta)=1-p e^{-\lambda_{1} \delta}-(1-p) e^{-\lambda_{2} \delta}$ (in green),

c) $G(\delta)=1-e^{-\lambda \delta}$ (in blue).

We choose the parameters $\lambda_{1}=5, \lambda_{2}=15, p=0.3$ and $\alpha=2$, the last one in order to make the gamma distribution IFR. Since we want the three distributions to have a same mean, the remaining parameters are determined as

$$
\lambda=\frac{\lambda_{1} \lambda_{2}}{\lambda_{1}(1-p)+p \lambda_{2}}, \quad \beta=\alpha \lambda
$$

Figure 4 Comparison among expected rewards associated with a same concave $\rho$ and different $G$ 's

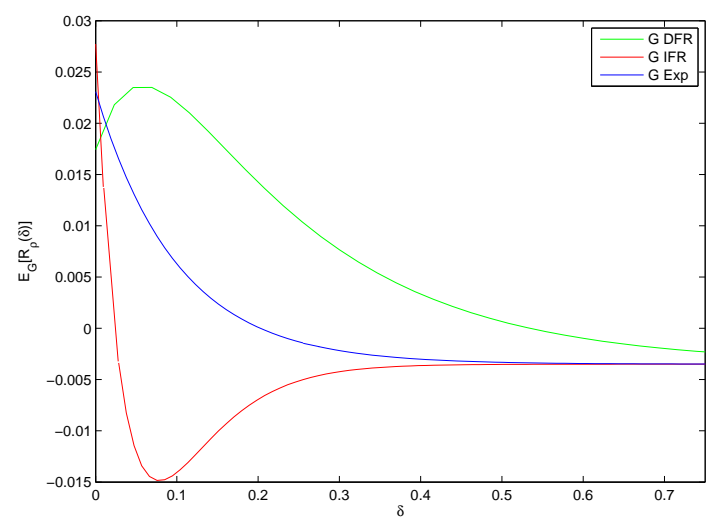

It is interesting now to compare the figure above with the analogous figure 2, corresponding to the case when $\rho$ is linear.

First we consider the green curves, corresponding to the DFR case. We notice that in the present case, the point of maximum is shifted towards right and the maximum is more pronounced.

In fact, in view of Lemma 2, heuristically speaking, Eq. (14) is more easily satisfied than $\alpha(\delta)>r(\delta)$. By an analogous argument, the red curve, corresponding to the IFR case, has a less pronounced minimum and takes higher values with respect to the linear case. 
The blue curves, corresponding to the no ageing case, are not substantially different, coherently with Corollary 3.

Finally, we show how, fixing $G$ and $(\tilde{k}, k, c)$, the reward function $\rho$ affects the shape of $\mathcal{R}$ and therefore the decision about the burn-in duration.

Finally, we turn to illustrate the influence of the reward function $\rho$ on the shape of the function $\mathcal{R}$. By looking at the figures 5 and 6 , we can focus some aspects of the role of $\rho$ in the solution of the optimal problem. On this purpose we compare, on a same figure, the graphs of $\mathcal{R}$ that are obtained for different forms of $\rho$, and for a fixed choice of $G$ and $(k, \tilde{k}, c)$.

Let us fix a DFR $G$ and let $\rho$ vary.

In Fig. 5, we consider $G(t)=1-p e^{-\lambda_{1} t}-(1-p) e^{-\lambda_{2} t}$, with $p=0.3, \lambda_{1}=5, \lambda_{2}=15$, and the cost parameters $(\tilde{k}, k, c)=(-0.2,-0.03,-0.0036)$. The $\mathcal{R}$ associated with a convex $\rho$ admits no local optima besides 0 . The function $\mathcal{R}$ associated with a linear $\rho$ admits a maximum point in $\delta^{*}$. The same also happens when $\rho$ is concave, with $\delta_{c v}^{*}>\delta^{*}$; however in this last case, the optimum is not significant, since its value is always negative.

Figure 5 Comparison among expected rewards associated with a same DFR $G$ and different $\rho$ 's

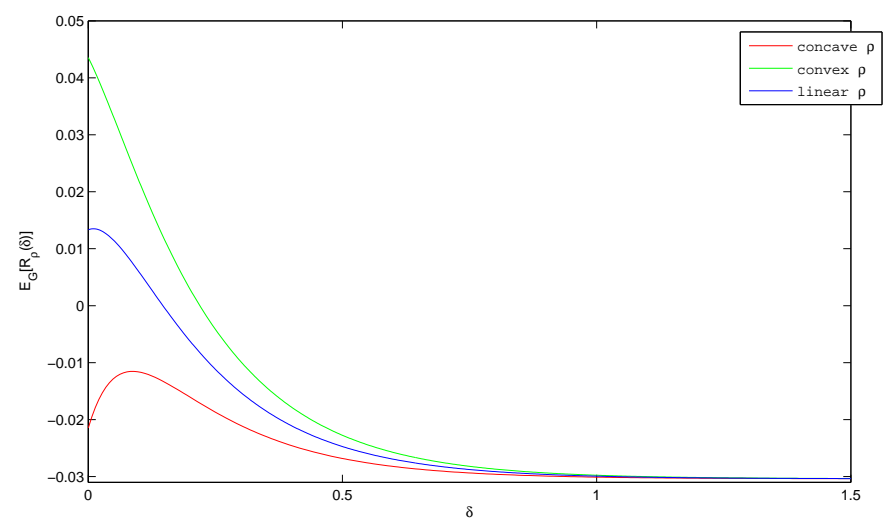

Figure 6 Comparison among expected rewards associated with a same DFR $G$ and different $\rho$ 's

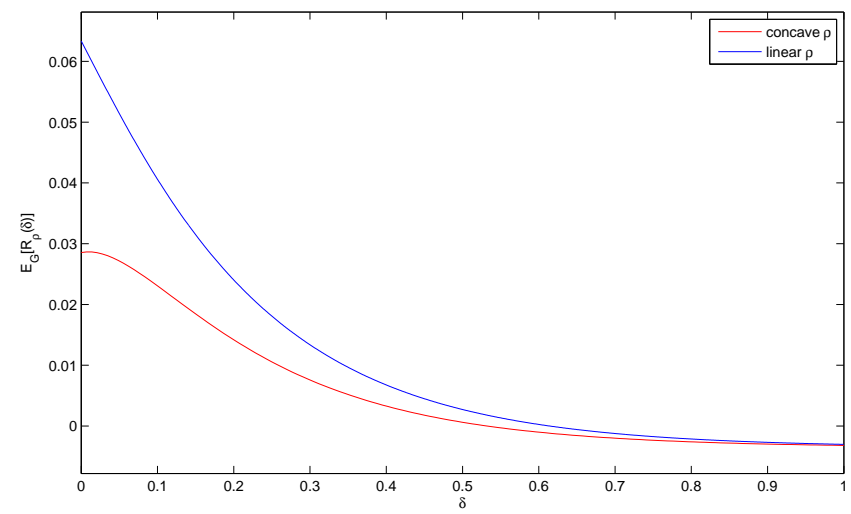

By considering instead (see Fig. 6)

$$
(\tilde{k}, k, c)=(-0.15,-0.003,-0.005),
$$




$$
G(t)=1-p e^{-\lambda_{1} t}-(1-p) e^{-\lambda_{2} t},
$$

with $p=0.3, \lambda_{1}=2, \lambda_{2}=5$, and let $\rho$ be concave or linear, we obtain significative values also correspondingly to the concave $\rho$ and a maximum point greater than 0 , but we lose the maximum for $\rho$ linear (cf Fig. 1).

\section{Discussion and concluding remarks}

After presenting some preliminary facts about notions of ageing and risk, we obtained some results about the optimal burn-in time problem, under some types of ageing conditions. This section will be devoted to shortly comment on different aspects of such arguments.

As first, we consider the circumstance that ageing properties of the lifetime - of the component to be tested - are not at all sufficient, by themselves, to establish the value of the optimal burn-in time. It is generally clear that the form of the reward function also plays a key role in the problem. In the previous sections, we in particular singled out some of the technical reasons why this phenomenon arises, at least under our choice of cost functions. In the linear case, the key role of the function $\rho$ is made evident, by Proposition 2 (see, more precisely, Eq. (7): for the existence of a strictly positive optimal burn-in time, the coefficient $\rho_{0}$ must satisfy, for some $\delta>0$, the conditions

$$
\left\{\begin{array}{l}
\rho_{0}>0 \\
\rho_{0}=c+(k-\widetilde{k}) r(\delta) .
\end{array}\right.
$$

In the non-linear case, Eq. (16) generalizes to

$$
\left\{\begin{array}{l}
\mathbb{E}\left[\rho^{\prime}(T-\delta) \mid T>\delta\right]>0, \\
\mathbb{E}\left[\rho^{\prime}(T-\delta) \mid T>\delta\right]=c+(k-\widetilde{k}) r(\delta),
\end{array}\right.
$$

that can be derived also from Theorem 2. A comparison between Eq.'s (16) and (17) readily highlights the probabilistic meaning of the constant $\rho_{0}$ appearing in Eq. (16): we must look at $\rho_{0}$ as

$$
\rho_{0}=\mathbb{E}\left[\rho^{\prime}(T-\delta) \mid T>\delta\right] .
$$

We notice moreover that, depending on the shape of $\rho$, also the ageing properties of $\bar{G}$ can have a different impact on the solution of the burn-in problem. In fact, in the linear case $\rho(t)=\rho_{0} t$, the condition $r^{\prime}(\delta)<0$ (this can be seen as a point-wise ageing property of $\bar{G}_{\delta}$ ) is both sufficient and necessary for $\delta$ being a locally optimal burn-in time. Still maintaining the condition $\rho(t)=\rho_{0} t$ and adding the stronger condition that $\bar{G}$ is IFR, DFR, bathtub or upside down bathtub, it can be furthermore proven that the optimal burn-in time is unique. A more precise statement and a formal proof can be found in Foschi (2011). On the contrary, when $\rho(t)$ is non-linear, Theorems 2 and 3 provide us with only sufficient conditions, linking the position of the optimal burn-in time to ageing properties of $\bar{G}$.

In view of the previous considerations, it is obvious that even the decision whether burn-in is to be implemented or not cannot be taken by only analyzing the ageing properties of $\bar{G}$. For instance, Proposition 2 and Theorem 2 provide us with some information about the solution of this decision problem, in the linear and non-linear case respectively. Notice that, in the non-linear case, the exact positions of the optima cannot be exactly recovered. However, our results allow us to give bounds for them, by means of inequalities based on relevant ageing properties of $\bar{G}$.

Concerning the search of the solution, we emphasize that our conditions can involve different aspects of $\bar{G}$. This fact depends on the choice of the cost structure, reflected by the function $R$. In correspondence with different functions $R$, also the functions involved in the conditions for optimality may be different. This issue is confirmed in the burn-in literature, where different functions have been considered as relevant, depending on the structure of the problem. Among these functions, we mainly have 
- the failure rate $r(t)$,

- the mean residual lifetime $\mu(t)=\mathbb{E}[T-t \mid T>t]$,

- the ratio $z(t)=\frac{g(t+\tau)}{g(t)}$.

As mentioned, the choice among such functions may explicitly depend on the reward function. We can see in particular that, under the reward function $(1), \mu(t)$ is relevant only in the case when $\rho$ is linear; in the general case $\mu(t)$ must be replaced by $\mathbb{E}[\rho(T-t) \mid T>t]$.

In those problems where a mission time (or a warranty time) is present, the form of the ratio $z(t)$ becomes relevant, instead of $r(t), \mu(t)$ or $\mathbb{E}[\rho(T-t) \mid T>t]$.

We might then argue that the form of $\mathcal{R}$ determines which are the distributional characteristics to be analyzed and, consequently, the ageing properties of interest.

On the other hand, the choice of the reward function is often influenced by the planned use of the units and by the expected types of failure. Then we can conclude, in ultimate analysis, that the reward function is not independent of the probabilistic model about lifetimes of the components and related ageing properties.

As well-known, different forms of the cost functions have been considered in the burn-in literature. The argument above points out then that the imposition of the cost function is not a matter of arbitrary choice. Rather, this choice depends on the planned usage of the components and it is, more or less explicitly, related to the probabilistic model for their lifetimes.

In any case, one can generally distinguish three different types of costs:

a) the cost due to the failure of a components during the operative life;

b) the cost of failures occurring during the burn-in procedure;

c) the costs due to the actual implementation of burn-in.

In some papers the cost due to the failure during the operative life also comprises the gain deriving from the usage of components and then it is (as a cost) a decreasing function of the failure time. In this paper we preferred to separate the pure cost due to failure from the gain produced by usage. Notice in fact the presence, within the reward function (1), of the sum of the two different addenda, of opposite sign, $\rho(t-\delta)$ and $\widetilde{k}$ in the term to be multiplied by $\mathbf{1}_{\{t>\delta\}}$.

As thoroughly discussed in Block and Savits (1997), some cost functions are directly built upon performance-based criteria. Namely one starts by considering as rewards different probability characteristic (such as expected values, survival probabilities, etc) of residual lifetimes of tested components; then some simple cost structure is added to these objects. We believe that our analysis of reward functions of the form (1), with $\rho$ non-linear, can add some new element of interest for further research on this topic.

In some other papers (see e.g. Perlstein et al. (2001)) the presence of a warranty period is considered in the definition of the cost function. This aspect goes beyond the analysis based on the family of reward functions in Eq. (1). Some related research might be of interest, however.

We furthermore notice that, in our derivations, we assumed that the cost of type b) has the simple form $k \mathbf{1}_{\{t<\delta\}}$. Provided some appropriate change is made, we also might extend our analysis to the case $k(t) \mathbf{1}_{\{t<\delta\}}$. The possibility of such an extension could in particular be useful in view of the ensuing considerations.

Several papers have been devoted to the analysis of burn-in for the case of several components with dependent, or in particular exchangeable, lifetimes. See e.g. Runggaldier (1993), Costantini and Spizzichino (1997), Perlstein et al. (2001), Jensen and Spizzichino (2004).

In this paper we limited however the analysis of the burn-in problem to the case of a single component.

This limitation is, anyway, rather common in the reliability literature and, as a concluding point of our discussion, we highlight some justifications in this respect. 
First, notice that the burn-in problem in the case of several components immediately reduces to a set of single-component burn-in problems, when the components' lifetimes are stochastically independent (the number of different problems is equal to the number of different distributions appearing among the marginal distributions of the components).

In a Bayesian point of view, the assumption of independence, even if actually very strong, may be accepted to some extent, according to the following considerations. As far as some stochastic dependence among components is present, we might exploit dependence in order to learn about the behaviour of a component on the basis of information obtained by testing different components. This circumstance may sometimes avoid burn-in. Consider in this respect that burn-in is, in ultimate analysis, an extreme form of learning about the behaviour of a component, obtained by testing the component itself for an initial period of its lifetime. We then could try to avoid burn-in if a different source of information could be available under the form of statistical experiments. In other words, we might consider burn-in as a last resource to be considered in face of a residual uncertainty, that remains when all other possibilities of learning has been exhausted (see also the discussion in Spizzichino (1993)).

However, the reduction to single-components burn-in procedures can be still justified even in the case of dependence among components, at least under the assumption of exchangeability.

Let us consider, in fact, the case when we have several exchangeable components tested simultaneously and the related problem of deciding whether continuing the burn-in or not and, in case, how long the residual burn-in should last for each component. It can be shown that such problem actually materializes only at the instants when some failure is observed. See in this respect considerations presented e.g. in Costantini and Spizzichino (1997), Jensen and Spizzichino (2004). From a purely probabilistic viewpoint this claim may be explained by taking into account that the time for stopping burn-in must be a stopping time with respect to the filtration defined by progressive observation of failures and by using a result (see e.g. (Bremaud 1981, Theorem T33)) about the structure of stopping times for a simple counting process.

Based on such a structure, one might argue as follows. Consider the burn-in problem for $n$ components with exchangeable lifetimes $T_{1}, \ldots, T_{n}$. Such a problem reduces to considering $n$ onecomponent burn-in problems that are respectively met at the instants $0, T_{(1)}, \ldots, T_{(n-1)}$ and that separately concern with the one-dimensional random variables $T_{(1)}, T_{(2)}-T_{(1)}, \ldots, T_{(n)}-T_{(n-1)}$. Here $T_{(1)}, \ldots, T_{(n)}$ denote of course the order statistics of $T_{1}, \ldots, T_{n}$ and the distribution to be considered for $T_{(k)}-T_{(k-1)}$ is the one that is obtained by conditioning on the information collected up to $T_{(k-1)}$. Such a procedure leads us to considering $n$ single-components burn-in problems where, however, the cost of a failure during burn-in may depend on the failure-instant in a complex way.

\section{Acknowledgements}

We thank two anonymous referees for criticism and comments that led us to improve a previous version of this paper. We also like to thank Prof. P. Delquié for pointing out the interest of the reference Bordley and LiCalzi (2000).

This work was partially supported by Italian MIUR (PRIN on Probability and Finance 2008) and Università La Sapienza (Research Project 2009 "Modelli e Algoritmi Stocastici: Convergenza ed Ottimizzazione").

\section{References}

Barlow, R. E. and Proschan, F. 1965. Mathematical theory of reliability, The SIAM series in applied mathematics, John Wiley \& sons, New York. 
Bebbington, M., C.-D. Lai, R. Zitikis. 2007. Optimum burn-in time for a bathtub-shaped failure distribution. Methodology and Computing in Applied Probability 9(1) 1-20. doi:10.1007/s11009-006-9001-7. URL http://dx.doi .org/10.1007/s11009-006-9001-7.

Block, H. W., T. H. Savits. 1997. Burn-in. Statistical Science 12(1) 1-19.

Bordley, R., M. LiCalzi. 2000. Decision analysis using targets instead of utility functions. Decis. Econ. Finance 23(1) 53-74. doi:10.1007/s102030050005.

Bremaud, P. 1981. Point processes and queues. Martingale dynamics. Springer Series in Statistics, SpringerVerlag, New York-Berlin.

Cha, J. H. 2001. Burn-in procedures for a generalized model. Journal of Applied Probability $38542-553$.

Clarotti, C. A., F. Spizzichino. 1990. Bayesian burn-in decision procedures. Prob. Eng. and Inform. Sc. 4 437-445.

Costantini, C., F. Spizzichino. 1997. Explicit solution of an optimal stopping problem: the burn-in of conditionally exponential components. J. Appl. Probab. 34(1) 267-282.

DeGroot, M. H. 2004. Optimal Statistical Decisions. Wiley Classics Library, Wiley.

Foschi, R., F. Spizzichino. 2008. Semigroups of semicopulas and evolution of dependence at increase of age. Mathware 8 Soft Computing XV(1) 95-111.

Foschi, R. 2011. Interval bounds for the optimal burn-in times for concave or convex reward functions. Preprint.

Glaser, R. E. 1980. Bathtub and related failure rate characterizations. Journal of the American Statistica Association 75(371) 667-672. Theory and Methods section.

Gupta, R. C. 2001. Nonmonotonic failure rates and mean residual life functions. Y. Hayakawa, T. Irony, M. Xie, eds., System and Bayesian Reliability, Series on Quality, Reliability and Engineering Statistics, vol. 5, chap. 9. World Scientific, New Jersey, 147-162.

Herberts, T., U. Jensen. 1999. Optimal stopping in a burn-in model. Commun. Statist. Stochastic Models 15 931-951.

Jensen, U., F. Spizzichino. 2004. The burn-in problem - a discussion of sequential stop and go strategies. Mathematical reliability: an expository perspective, Internat. Ser. Oper. Res. Management Sci., vol. 67. Kluwer Acad. Publ., Boston, MA, 207-229.

Kuo, W., Y. Kuo. 1983. Facing the headaches of early failures: a state-of-the-art review of burn-in decisions. Proceedings of the IEEE, vol. 71. 1257-1266.

Lai, C.-D. and Xie, M. 2004. Stochastic Ageing and Dependence for Reliability. Springer, New York.

Lynn, N. J., N. D. Singpurwalla. 1997. Comment: "burn-in" makes us feel good. Statistical Science 12 13-19.

Mi, J. 1994. Burn-in and maintenance policies. Advances in Applied Probability 26 207-221.

Perlstein, D., Jarvis, W. H., Mazzuchi, T. A. 2001. Bayesian calculation of cost-optimal burn-in test duration for the mixed exponential populations. Journal of Reliability Engineering and System Safety 72 265273.

Runggaldier, W. J. 1993. On Stochastic Control Concepts for Sequential Burn-in Procedures. In RELIABILITY AND DECISION MAKING (R.E.Barlow, C.A.Clarotti, F.Spizzichino, eds.), Chapman \& Hall, London, 211-232.

Scarsini, M. 1994. Comparing Risk and Risk Aversion. In Stochastic orders and Their Applications (Shaked, M. and Shanthikumar, J. G., Probability and Mathematical Statistics, chap. 12. Academic Press, Boston, 351-378.

Singpurwalla, N. D. 2006. Reliability and risk. Wiley Series in Probability and Statistics, John Wiley \& Sons Ltd., Chichester. doi:10.1002/9780470060346. URL http://dx.doi.org/10.1002/9780470060346. A Bayesian perspective.

Spizzichino, F. 1993. A unifying model for the optimal design of life-testing and burn-in. Reliability and decision making (Siena, 1990). Chapman \& Hall, London, 189-210. 